\title{
A one-dimensional liquid of fermions with tunable spin
}

\author{
Guido Pagano ${ }^{1,2}$, Marco Mancini ${ }^{1,3}$, Giacomo Cappellini ${ }^{1}$, Pietro Lombardi ${ }^{1,3}$, Florian Schäfer ${ }^{1}$, \\ Hui Hu ${ }^{4}$, Xia-Ji Liu ${ }^{4}$, Jacopo Catani ${ }^{1,5}$, Carlo Sias ${ }^{1,5}$, Massimo Inguscio ${ }^{1,3,5}$ and Leonardo Fallani ${ }^{1,3,5 \star}$
}

\begin{abstract}
Correlations in systems with spin degree of freedom are at the heart of fundamental phenomena, ranging from magnetism to superconductivity. The effects of correlations depend strongly on dimensionality, a striking example being one-dimensional (1D) electronic systems, extensively studied theoretically over the past fifty years ${ }^{1-7}$. However, the experimental investigation of the role of spin multiplicity in 1D fermions-and especially for more than two spin components-is still lacking. Here we report on the realization of $1 \mathrm{D}$, strongly correlated liquids of ultracold fermions interacting repulsively within $\operatorname{SU}(N)$ symmetry, with a tunable number $\boldsymbol{N}$ of spin components. We observe that static and dynamic properties of the system deviate from those of ideal fermions and, for $N>2$, from those of a spin-1/2 Luttinger liquid. In the large- $N$ limit, the system exhibits properties of a bosonic spinless liquid. Our results provide a testing ground for many-body theories and may lead to the observation of fundamental 1D effects ${ }^{8}$.
\end{abstract}

One-dimensional quantum systems show specific, sometimes counterintuitive behaviours that are absent in the 3D world. These behaviours, predicted by many-body models of interacting bosons ${ }^{9}$ and fermions ${ }^{2-4}$, include the 'fermionization' of bosons ${ }^{10}$ and the separation of spin and density (most commonly referred to as 'charge') branches in the excitation spectrum of interacting fermions. The last phenomenon is predicted within the celebrated Luttinger liquid model ${ }^{5}$, which describes the low-energy excitations of interacting spin-1/2 fermions. Although the Luttinger approach describes qualitatively the physics of a number of $1 \mathrm{D}$ systems ${ }^{11,12}$, the problem of how to extend it to a more detailed description of real systems has puzzled physicists over the years? ${ }^{7}$. In this exploration the physics of spin has played a key role.

Ultracold atoms have proved to be a precious resource to study 1D physics, as they afford exceptional control over experimental parameters. Most of the experiments so far have been performed with spinless bosons, which for instance led to the realization of a Tonks-Girardeau gas ${ }^{13,14}$. On the other hand, 1D ultracold fermions are a promising system to observe a number of elusive phenomena, such as Stoner's itinerant ferromagnetism ${ }^{15}$ and the physics of spin-incoherent Luttinger liquids 6 . However, only a few pioneering works, dealing with spin-1/2 particles $^{16-18}$, have been reported so far.

In parallel, ultracold two-electron atoms have been recently proposed for the realization of large-spin systems with $\mathrm{SU}(N)$ interaction symmetry ${ }^{19,20}$, and the first experimental investigations have been reported ${ }^{21}$. This novel platform enables the simulation of
1D systems with a high degree of complexity, including spin-orbitcoupled materials ${ }^{22}$ or SU $(N)$ Heisenberg and Hubbard chains ${ }^{23,24}$. Moreover, the investigation of these multi-component fermions is relevant for the simulation of field theories with extended $\mathrm{SU}(N)$ symmetries ${ }^{25}$.

In this Letter we report on the realization of $1 \mathrm{D}$ quantum wires of repulsive fermions with a tunable number of spin components, which are created by tightly trapping ultracold ${ }^{173} \mathrm{Yb}$ atoms in a 2D optical lattice (Fig. 1a). The purely nuclear spin $I=5 / 2$ of ${ }^{173} \mathrm{Yb}$ results both in the independence of the interaction strength from the nuclear spin state and in the absence of spin-changing collisions. The latter feature is particularly important for our experiments, as it implies the stability of any spin mixture. The atoms experience an axial harmonic confinement with (angular) frequency $\omega_{x} \approx 2 \pi \times 80 \mathrm{~Hz}$ and a strong radial confinement with $\omega_{\perp}=2 \pi \times 25 \mathrm{kHz}$, resulting in the occupation of the radial ground state. We use optical spin manipulation and detection techniques (see Supplementary Information) to prepare the system in an arbitrary number $N \leq 2 I+1=6$ of spin components (Fig. 1b), thus realizing different $\mathrm{SU}(N)$ symmetries. We directly compare systems with different $N$, keeping the atom number per spin component $N_{\mathrm{at}} \simeq 6,000(\approx 20$ atoms per spin component in the central wire $)$ and all the other parameters constant. This approach enables us to examine how the physics of a strongly-interacting $1 \mathrm{D}$ fermionic system changes as a function of $N$.

\section{Momentum distribution}

We investigate the correlations in the $1 \mathrm{D}$ wires by observing the momentum distribution $n(k)$ ( $k$ is the momentum divided by the reduced Planck's constant $\hbar$ ). We measure this quantity by extinguishing the trapping light and imaging the atomic cloud after a ballistic expansion, as done in previous works to measure $n(k)$ of a Tonks-Girardeu gas ${ }^{13}$. A typical image is reported in Fig. $2 c$, where $\hat{x}$ denotes the wire axis. Integration over $\hat{y}$ results in the $n(k)$ curves plotted in Fig. 2a for different $N$ (the curves are normalized to have the same unit area). In the non-interacting case $N=1$ the data (solid blue) are very well accounted for by the theory of a trapped ideal Fermi gas (dashed blue, see Supplementary Information). Increasing $N$, we observe a clear monotonic broadening of $n(k)$, with a reduction of the weight at low $k$ and a slower decay of the large- $k$ tails.

The observed $n(k)$ broadening arises from a pure effect of correlations that goes beyond standard mean-field physics. To give a qualitative understanding of this phenomenon, we consider

\footnotetext{
${ }^{1}$ LENS European Laboratory for Nonlinear Spectroscopy, Sesto Fiorentino 50019, Italy, ${ }^{2}$ Scuola Normale Superiore di Pisa, Pisa 56126, Italy, ${ }^{3}$ Department of Physics and Astronomy, University of Florence, Sesto Fiorentino 50019, Italy, ${ }^{4}$ Centre for Atom Optics and Ultrafast Spectroscopy, Swinburne University of Technology, Melbourne 3122, Australia, 5 INO-CNR Istituto Nazionale di Ottica del CNR, Sezione di Sesto Fiorentino, Sesto Fiorentino 50019 , Italy. *e-mail: fallani@lens.unifi.it
} 


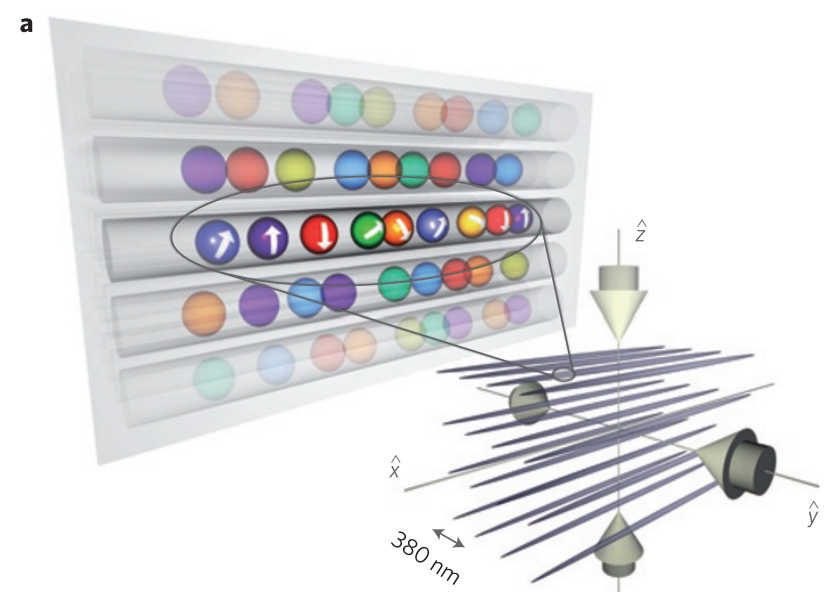

b
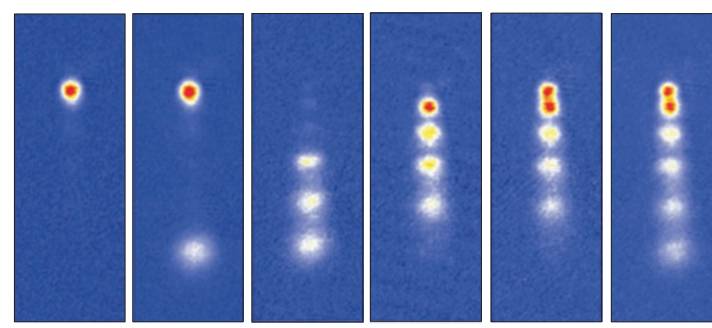

$+5 / 2$

$+1 / 2$

$-1 / 2$

$-3 / 2$

$-5 / 2$

2

Figure 1 | Ultracold 1D fermionic liquids with tunable spin. a, A 2D optical lattice is used to create an array of independent quantum wires of ultracold ${ }^{173} \mathrm{Yb}$ with six possible nuclear spin orientations. $\mathbf{b}$, The nuclear spin of the atoms can be manipulated with optical pumping techniques, resulting in a tunable number of spin components, and analysed with optical Stern-Gerlach detection (see Supplementary Information).

spin-1/2 fermions with infinite repulsion. In this limit, the density-density correlation function $G_{\uparrow \downarrow}(d)=\left\langle\hat{n}_{\uparrow}(x+d) \hat{n}_{\downarrow}(x)\right\rangle$ (where $\hat{n}_{\uparrow}(x)$ and $\hat{n}_{\downarrow}(x)$ are the density operators for the two spin components) falls to zero for $d \rightarrow 0$ as $G_{\uparrow \uparrow}(d)$ does in the case of a spin-polarized gas, thus mimicking the effects of Pauli repulsion between distinguishable particles. This 'fermionization', restricting the effective space which is available to the particles, causes them to populate states with larger momentum ${ }^{26,27}$. We note that an opposite behaviour would be predicted by a mean-field treatment of interactions neglecting correlations between trapped fermions: the effectively weaker confinement along $\hat{x}$ induced by the atom-atom repulsion would lead to more extended single-particle wavefunctions, hence to a decreased width of $n(k)$ (Fig. 2b). For $N=2$ the interaction regime of our 1D samples is described by the parameters $\gamma \simeq 4.8$ and $K \simeq 0.73$ (see Supplementary Information), lying in the strongly-correlated regime between the ideal Fermi gas $(\gamma=0, K=1)$ and a fully fermionized gas $(\gamma=\infty, K=0.5)$.

The details of $n(k)$ depend nontrivially on the temperature, owing to the thermal population of spin excitations. The temperature regime for our experiments, $T \simeq 0.3 T_{\mathrm{F}}$ (where $T_{\mathrm{F}}$ is the Fermi temperature), is slightly below the predicted temperature scale $T_{\mathrm{S}} \simeq 0.4 T_{\mathrm{F}}$ for spin excitations (see Supplementary Information), in the crossover between the spin-ordered regime for $T \ll T_{\mathrm{S}}$ and that of a spin-incoherent Luttinger liquid for $T \gg T_{\mathrm{S}}$ (ref. 6). Figure $2 \mathrm{~b}$ shows the theoretical $n(k)$ for $N=2$ and infinite repulsion in the limiting regimes $T=0$ and $T \gg T_{\mathrm{S}}$ (light and dark solid curves, derived from refs 26 and 27, respectively). Although both curves show an evident $n(k)$ broadening, in accordance with our observations, their shape is different and can be explained in terms a

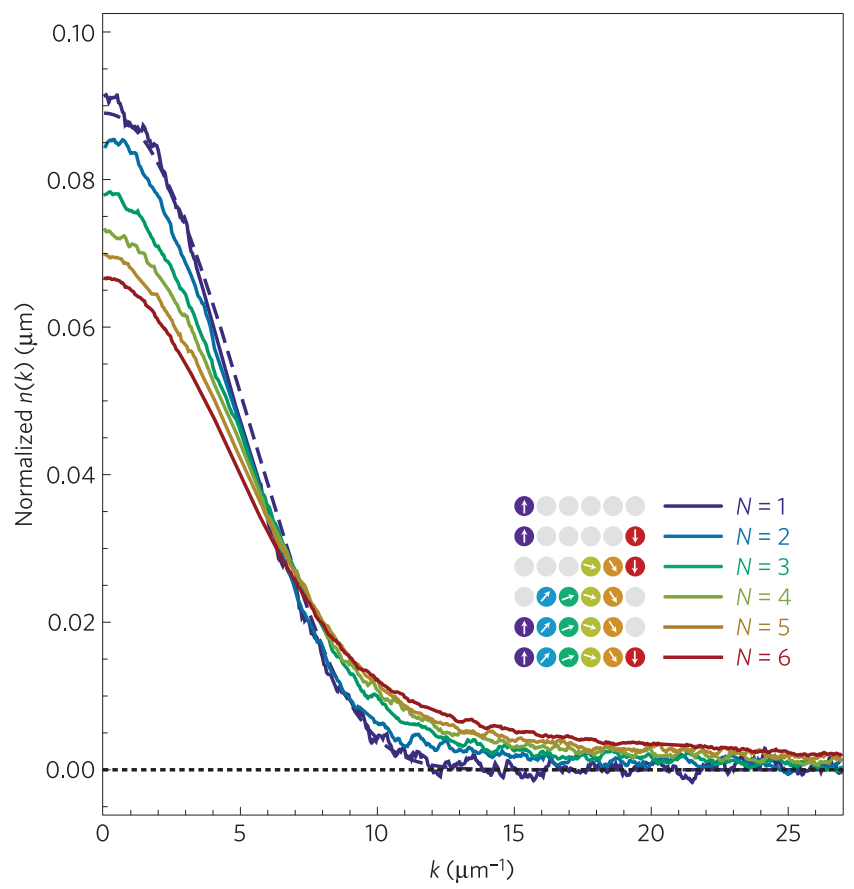

b
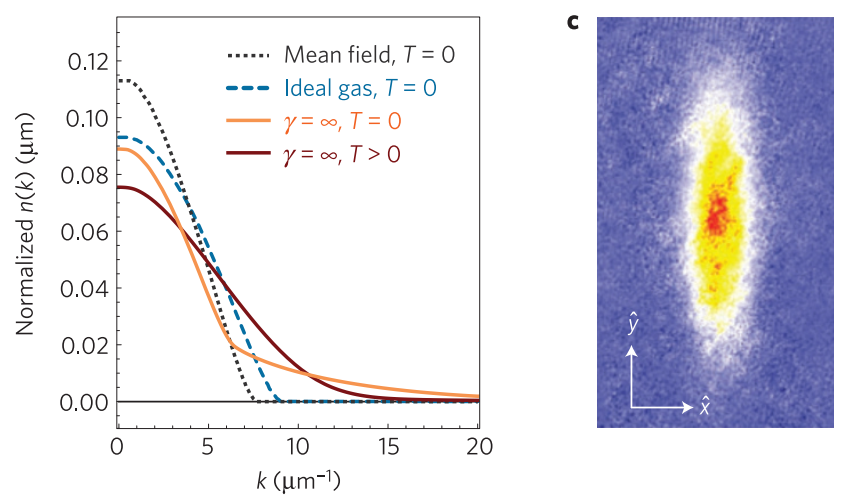

Figure 2 | Momentum distribution of the 1D fermions. a, Solid lines: momentum distribution $n(k)$ measured with time-of-flight absorption imaging for different $N$ and the same atom number $N_{\text {at }}$ per spin component (each curve results from the average of 30-50 experimental images, after integration along the $\hat{y}$ axis and normalization to unity area, i.e. $\left.\int \mathrm{n}(\mathrm{k}) \mathrm{dk}=1\right)$. Dashed line: theoretical curve for $N=1$ based on the ideal trapped Fermi gas theory, after averaging over the inhomogeneous distribution of atoms in the different wires. $\mathbf{b}$, Theoretical $n(k)$ for the $N=2$ system derived from different models (see Supplementary Information): ideal Fermi gas at $T=0$ (dashed), mean-field treatment of finite interactions at $T=0$ (dotted), full many-body problem for infinite interactions both for $T=0$ (light solid, from ref. 26) and $T_{\mathrm{S}} \ll T \ll T_{\mathrm{F}}$ (dark solid, from ref. 27). Whereas the mean-field curve shows a different behaviour from the one observed in the experiment, the many-body curves account for the observed broadening. c, Averaged absorption image (the $\hat{x}$ axis denotes the direction of the wires).

of a modified effective Fermi momentum ${ }^{28}$. Exact calculations for finite interactions and finite temperatures are challenging, thus making our system a profitable quantum simulation resource for the fundamental problem of $1 \mathrm{D}$ interacting fermions.

\section{Probing excitations}

A distinctive feature of $1 \mathrm{D}$ fermions is the existence of a well-resolved excitation spectrum at small momenta $\hbar q \ll \hbar k_{\mathrm{F}}$ (where $k_{\mathrm{F}}$ is the Fermi wave vector). Number-conserving excitations 
a

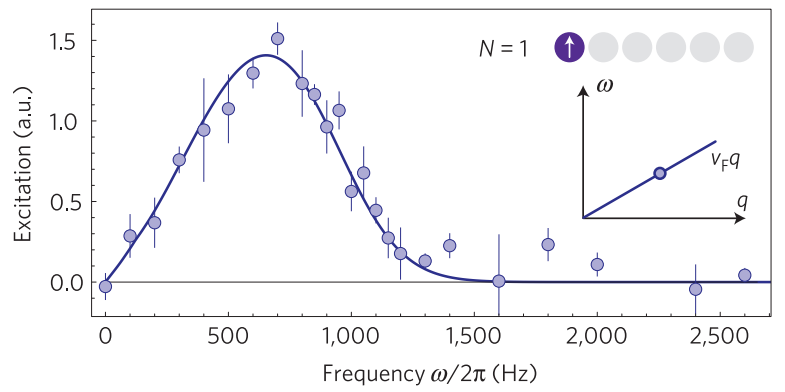

b
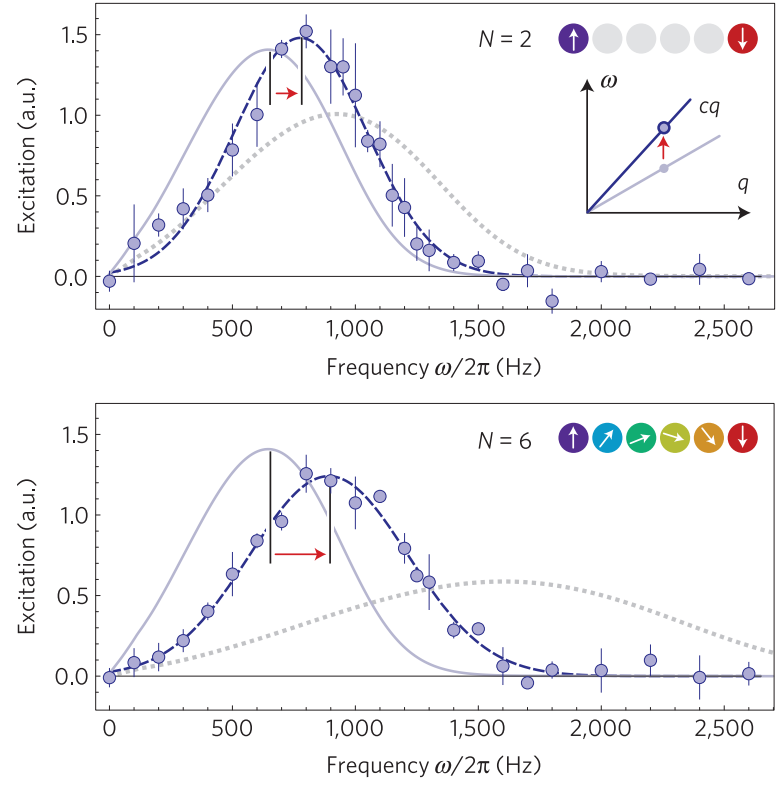

Figure 3 | Excitation spectra of the 1D fermions. a-c, The points show the measured increase in atomic momentum transfer after a Bragg excitation with energy $\hbar \omega$ and momentum $\hbar q \simeq 0.2 \hbar k_{\mathrm{F}}^{0}$ (see text) for $N=1$ (a), $N=2$ (b) and $N=6$ (c) spin components and the same atom number $N_{\text {at }}$ per spin component. The error bars are standard deviations over up to five repeated measurements per frequency. The solid line is the calculated response function for the ideal Fermi gas $N=1$, while the dotted lines show the calculation in the limit of infinite repulsion. The dashed lines are Gaussian fits to the experimental points, to guide the eye and to extract the peak excitation frequency. Both the experimental and theoretical spectra have been normalized to unit area. The graphs in the inset show a sketch of the excitation spectrum at low $q$ for the ideal Fermi gas (a) and for the two-component Luttinger liquid (b) with repulsive interactions. The red arrows indicate the shift in the excitation resonance.

in the ideal 1D Fermi gas correspond to particle-hole pairs with energy $\hbar \omega=v_{\mathrm{F}} \hbar q$, where $v_{\mathrm{F}}=\hbar k_{\mathrm{F}} / m$ is the Fermi velocity (Fig. 3a, inset). This physical picture changes in the case of an interacting spin mixture, as excitations acquire a purely collective nature. According to the Luttinger theory, the spectrum of phononic excitations is still described by a linear dispersion $\omega=c q$, where $c=v_{\mathrm{F}} / K$ is a renormalized sound velocity ${ }^{1}$. In a two-component Luttinger liquid with contact repulsion one has $0.5<K<1$. This yields a sound velocity that is larger than $v_{\mathrm{F}}$ (Fig. $3 \mathrm{~b}$, inset), corresponding to an increased stiffness of the many-body state.

We have characterized the excitations of the fermionic wires by performing Bragg spectroscopy. This technique, relying on inelastic light scattering, allows the selective excitation of density waves with energy $\hbar \omega$ and momentum $\hbar q$ (see Supplementary Information). Figure $3 \mathrm{a}$ shows the measured spectrum for $N=1$ at low momentum transfer $\hbar q \simeq 0.2 \hbar k_{\mathrm{F}}^{0}$ (with $k_{\mathrm{F}}^{0}$ being the peak Fermi wave vector in the central wire). A clear resonance is

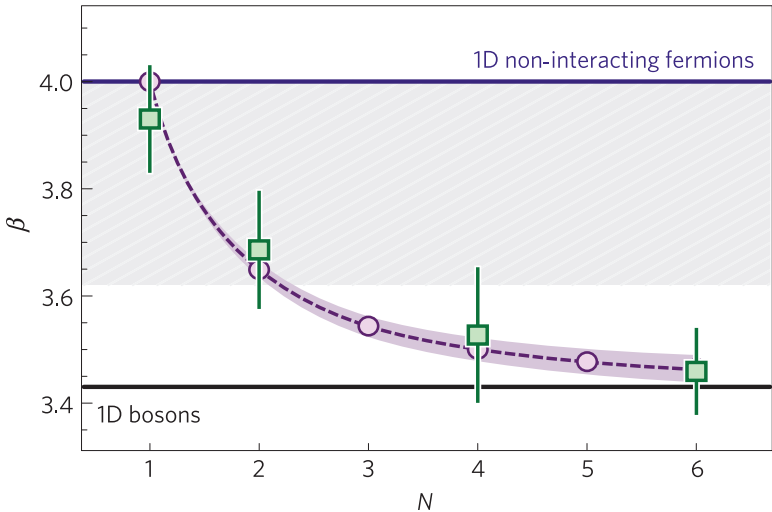

b

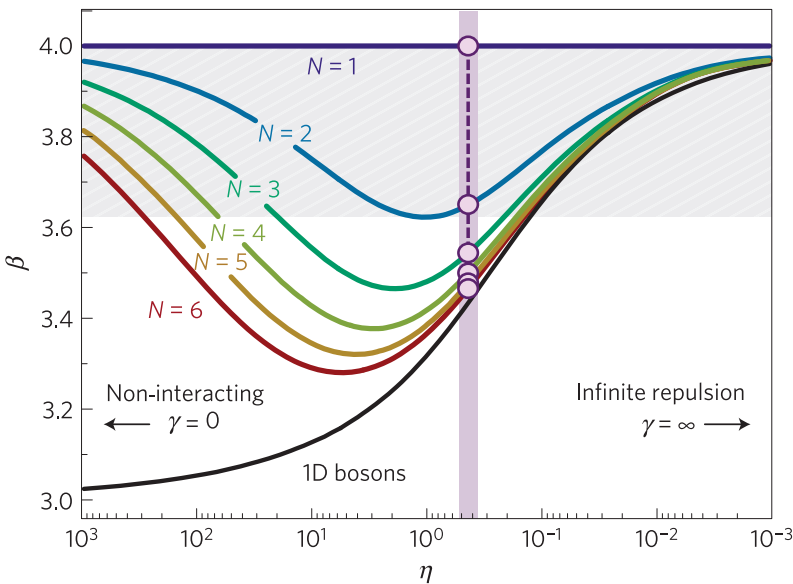

Figure 4 | Breathing oscillations. The quantity that is plotted in the graphs is the squared ratio $\beta=\left(\omega_{B} / \omega_{x}\right)^{2}$ of the breathing frequency $\omega_{B}$ to the trap frequency $\omega_{x} \cdot \mathbf{a}$, The squares show the experimental data, as a function of $N$, obtained as the weighted mean over sets of up to nine repeated measurements (the error bars indicate the standard deviation of the weighted mean). The circles show the theoretical predictions for the average interaction parameter (defined in the text) $\eta=0.44$ for our experiment. The dashed line is a guide to the eye, while the height of the violet shaded region indicates the uncertainty on the theoretical values resulting from the experimental uncertainty $\Delta \eta=0.08$ (coming from the measured atom number and trapping frequencies). The upper horizontal line shows the theoretical value for the non-interacting Fermi gas $(N=1)$, while the lower line shows the result for 1D spinless bosons. $\mathbf{b}$, The lines show the theoretical dependence of $\beta$ on the interaction parameter $\eta$. The circles show the predicted values for our average interaction parameter (also shown in $\mathbf{a}$ ), while the width of the violet shaded region indicates the experimental uncertainty $\Delta \eta=0.08$. In both panels the height of the grey region shows the range of $\beta$ for $N=2$ and any possible value of the repulsion strength.

observed, in excellent agreement with the calculated response for ideal fermions (solid line, with no free parameters). For $N=2$ the resonance is clearly shifted towards higher frequencies (Fig. 3b), as expected from the Luttinger theory. The measured shift $(+15 \pm 4) \%$ agrees with the expected $(+10 \pm 2) \%$ shift in the sound velocity predicted on the basis of the Luttinger theory for a trapped system (see Supplementary Information). For $N=6$ the spectrum shows a much larger shift $(+33 \pm 4) \%$ (Fig. 3c), which disagrees with the predictions for $N=2$, signalling an increased effect of interactions, in qualitative accordance with the $n(k)$ change of Fig. 2. We also plot the calculated spectra for trapped fermions with infinite interactions (Fig. 3b,c, dotted lines), which shows how the measured spectra lie between the response of the ideal Fermi gas and that of a fermionized system. 


\section{Collective mode frequencies}

More insight into the physics of multicomponent 1D fermions can be gained by studying low-energy breathing oscillations in which the cloud radius oscillates in time. We measure the frequency of this collective mode by suddenly changing the trap frequency and measuring the time evolution of the radius. In Fig. $4 \mathrm{a}$ we plot the measured squared ratio $\beta=\left(\omega_{\mathrm{B}} / \omega_{x}\right)^{2}$ of the breathing frequency $\omega_{\mathrm{B}}$ to the trap frequency $\omega_{x}$ as a function of $N$ (squares). For $N=1$ the measured value is in good agreement with the expected value $\beta=4$ for ideal fermions (upper horizontal line). With increasing $N$ our data clearly show a monotonic decrease of $\beta$, induced by the repulsive interactions in the spin mixture.

The dependence of $\beta$ on the interaction strength is remarkably nontrivial, already for $N=2$, as first predicted in ref. 29. Indeed, $\beta=4$ in both the limiting cases of an ideal gas $(\gamma=0)$ and a fermionized $(\gamma=\infty)$ system, whereas for finite repulsion it is expected to exhibit a nonmonotonic behaviour, with a minimum at finite interaction strength. The theoretical curves in Fig. $4 \mathrm{~b}$ show the expected dependence of $\beta$ on the interaction parameter $\eta=N_{\mathrm{at}}^{1}\left(a_{1 \mathrm{D}} / a_{x}\right)^{2}$ (where $N_{\mathrm{at}}^{1}$ is the number of atoms per wire, $a_{1 \mathrm{D}}$ is the $1 \mathrm{D}$ scattering length and $a_{x}$ is the trap oscillator length). We have derived these results by combining a Bethe Ansatz approach with the exact solution of the hydrodynamic equations describing a 1D fermionic liquid with $N$ components (see Supplementary Information). As $N$ is increased, the curves exhibit an increasingly larger redshift of $\beta$, and for $N \rightarrow \infty$ they asymptotically approach the curve for $1 \mathrm{D}$ spinless bosons. The circles indicate the theoretical values for the average $\eta=0.44$ in our experiment. The agreement between experiment and theory is excellent, as shown in Fig. 4a (we note that for $N=2$ both theory and experiment agree with the results of ref. 29).

The experimental data, accompanied by our theoretical curves, clearly show that changing $N$ causes markedly different effects from those induced by simply changing the interaction strength in an $N=2$ mixture. In fact, by increasing $N$, the constraints of the Pauli principle become less stringent and the number of binary-collisional partners increases, causing the system to acquire a more 'bosonic' behaviour. Our experimental value at $N=6$ clearly falls out of the range of $\beta$ expected for an $N=2$ liquid (Fig. 4, grey regions), and already approaches the value expected for $1 \mathrm{D}$ spinless bosons. This bosonic limit for $N \rightarrow \infty$ is a remarkable property of multi-component $1 \mathrm{D}$ fermions that has been pointed out theoretically only very recently ${ }^{30}$ and that our experimental system is capable to clearly evidence.

\section{Concluding remarks}

The possibility of tuning the number of spin components allows us to study different regimes of interplay between Fermi statistics and the degree of distinguishability in this novel 1D system. From a quantum simulation perspective, this realization provides a powerful test bench for large-spin models and opens a route towards the investigation of fundamental effects ranging from spin dynamics to novel magnetic phases.

\section{Received 26 August 2013; accepted 17 December 2013;} published online 2 February 2014

\section{References}

1. Giamarchi, T. Quantum Physics in One Dimension (Oxford Univ. Press, 2004)

2. Yang, C. N. Some exact results for the many-body problem in one dimension with repulsive delta-function interaction. Phys. Rev. Lett. 19, 1312-1315 (1967).

3. Gaudin, M. Un systeme a une dimension de fermions en interaction. Phys. Lett. A 24, 55-56 (1967).

4. Sutherland, B. Further results for the many-body problem in one dimension. Phys. Rev. Lett. 20, 98-100 (1968).

5. Voit, J. One-dimensional Fermi liquids. Rep. Prog. Phys. 58, 977-1116 (1995).

6. Fiete, G. A. The spin-incoherent Luttinger liquid. Rev. Mod. Phys. 79, 801-820 (2007).
7. Imambekov, A., Schmidt, T. L. \& Glazman, L. I. One-dimensional quantum liquids: Beyond the Luttinger liquid paradigm. Rev. Mod. Phys. 84, 1253-1306 (2012).

8. Recati, A., Fedichev, P. O., Zwerger, W. \& Zoller, P. Spin-charge separation in ultracold quantum gases. Phys. Rev. Lett. 90, 020401 (2003).

9. Lieb, E. H. \& Liniger, W. Exact analysis of an interacting Bose gas. I. The general solution and the ground state. Phys. Rev. 130, 1605-1616 (1963).

10. Girardeau, M. Relationship between systems of impenetrable bosons and fermions in one dimension. J. Math. Phys. 1, 516-523 (1960).

11. Bockrath, M. et al. Luttinger-liquid behaviour in carbon nanotubes. Nature 397, 598-601 (1999).

12. Yacoby, A. et al. Nonuniversal conductance quantization in quantum wires. Phys. Rev. Lett. 77, 4612-4615 (1996).

13. Paredes, B. et al. Tonks-Girardeau gas of ultracold atoms in an optical lattice. Nature 429, 277-281 (2004).

14. Kinoshita, T., Wenger, T. \& Weiss, D. S. Observation of a one-dimensional Tonks-Girardeau gas. Science 305, 1125-1128 (2004).

15. Cui, X. \& Ho, T. L. Ground-state ferromagnetic transition in strongly repulsive one-dimensional Fermi gases. Preprint at http://arxiv.org/abs/1305.6361 (2013).

16. Moritz, H., Stöferle, T., Günter, K., Köhl, M. \& Esslinger, T. Confinement induced molecules in a 1D Fermi gas. Phys. Rev. Lett. 94, 210401 (2005).

17. Liao, Y-A. et al. Spin-imbalance in a one-dimensional Fermi gas. Nature 467, 567-569 (2010).

18. Zürn, G. et al. Fermionization of two distinguishable fermions. Phys. Rev. Lett. 108, 075303 (2012).

19. Cazalilla, M. A., Ho, A. F. \& Ueda, M. Ultracold gases of ytterbium: Ferromagnetism and Mott states in an SU(6) Fermi system. New J. Phys. 11, 103033 (2009).

20. Gorshkov, A. V. et al. Two-orbital SU(N) magnetism with ultracold alkaline-earth atoms. Nature Phys. 6, 289-295 (2010).

21. Taie, S., Yamazaki, R., Sugawa, S. \& Takahashi, Y. An SU(6) Mott insulator of an atomic Fermi gas realized by large-spin Pomeranchuk cooling. Nature Phys. 8, 825-830 (2012).

22. Frischmuth, B., Mila, F. \& Troyer, M. Thermodynamics of the one-dimensional SU(4) symmetric spin-orbital model. Phys. Rev. Lett. 82, 835-838 (1999).

23. Bonnes, L., Hazzard, K. R. A., Manmana, S. R., Rey, A. M. \& Wessel, S. Adiabatic loading of one-dimensional $\mathrm{SU}(N)$ alkaline-earth-atom fermions in optical lattices. Phys. Rev. Lett. 109, 205305 (2012).

24. Messio, L. \& Mila, F. Entropy dependence of correlations in one-dimensional SU(N) antiferromagnets. Phys. Rev. Lett. 109, 205306 (2012).

25. Banerjee, D. et al. Atomic quantum simulation of $\mathrm{U}(\mathrm{N})$ and $\mathrm{SU}(N)$ non-Abelian lattice gauge theories. Phys. Rev. Lett. 110, 125303 (2013).

26. Ogata, M. \& Shiba, H. Bethe-ansatz wave function, momentum distribution, and spin correlation in the one-dimensional strongly correlated Hubbard model. Phys. Rev. B 41, 2326-2338 (1990).

27. Cheianov, V. V., Smith, H. \& Zvonarev, M. B. Low-temperature crossover in the momentum distribution of cold atomic gases in one dimension. Phys. Rev. A 71, 033610 (2005).

28. Feiguin, A. E. \& Fiete, G. A. Spectral properties of a spin-incoherent Luttinger liquid. Phys. Rev. B 81, 075108 (2010).

29. Astrakharchik, G. E., Blume, D., Giorgini, S. \& Pitaevskii, L. P. Interacting fermions in highly elongated harmonic traps. Phys. Rev. Lett. 93, 050402 (2004).

30. Yang, C. N. \& You, Y-Z. One-dimensional $w$-component fermions and bosons with repulsive delta function interaction. Chin. Phys. Lett. 28, 020503 (2011).

\section{Acknowledgements}

We would like to thank all the members of the Ultracold Quantum Gases group in Florence for fruitful discussions. We are indebted to P. Calabrese for enlightening discussions on the physics of 1D quantum systems, and to P. Cancio, G. Giusfredi and P. De Natale for early valuable contributions to the experimental set-up. We also thank M. Dalmonte A. Recati and M. Polini for valuable discussions. This work has been financially supported by IIT Seed Project ENCORE, ERC Advanced Grant DISQUA, EU FP7 Integrated Projects AQUTE and SIQS, MIUR Project PRIN2009, ARC Discovery Projects (Grants No. DP0984522 and No. DP0984637) and NFRP-China (Grant No. 2011CB921502).

\section{Author contributions}

All authors contributed to the writing of the manuscript. G.P., M.M., G.C., P.L., F.S., J.C., C.S., M.I. and L.F. built the experimental set-up, performed the measurements and analysed the data. H.H. and X-J.L. carried out the theoretical derivation of the breathing frequencies.

\section{Additional information}

Supplementary information is available in the online version of the paper. Reprints and permissions information is available online at www.nature.com/reprints.

Correspondence and requests for materials should be addressed to L.F.

\section{Competing financial interests}

The authors declare no competing financial interests. 